\title{
Pflegeroboter: Analyse und Bewertung aus Sicht pflegerischen Handelns und ethischer Anforderungen
}

\author{
Hartmut Remmers
}

\begin{abstract}
Zusammenfassung
In Zukunft ist ein wachsender Einsatz von Pflegerobotern zu erwarten, denen möglicherweise zwei Hauptfunktionen zugeschrieben werden können: Beitrag zur Verringerung einer sich ausweitenden Personallücke in der Pflege; Erleichterung der Pflege hinsichtlich physischer und psychischer Arbeitsbelastungen, teilweise auch durch Substitution originär pflegerischer Teilaufgaben im Bereich der Ernährung, Medikation, Aktivierung/Mobilisierung. Aus fachwissenschaftlicher Sicht stellen sich dabei Fragen, inwieweit und in welchem Ausmaße originär pflegerische Tätigkeiten wie die der persönlichen, vertrauensbildenden Beziehungsarbeit unterstützt/verbessert werden können oder (teil-)ersetzt werden sollen. Aus ethischer Perspektive stellen sich damit zusammenhängende Fragen, inwieweit durch den Einsatz von Robotern die Personalität des Menschen in verschiedenen Facetten seiner auch leiblich verstandenen Rezeptivität und seiner Spontaneität gegenseitigen Ausdruckshandelns und Ausdrucksverstehens gewahrt bleibt.
\end{abstract}

\subsection{Einleitung}

Durch den demografischen Wandel ergeben sich drei große Herausforderungen für den Pflegebereich: 1) die Zunahme eines wegen wachsender Hochaltrigkeit (Stichwort: altersgewandelte Gesellschaft) ebenso zunehmenden sozialen und gesundheitlichen

\footnotetext{
H. Remmers $(\square)$

Fachbereich Humanwissenschaften, Abteilung Pflegewissenschaft, Universität Osnabrück, Osnabrück, Deutschland

E-Mail: remmers@uni-osnabrueck.de

(C) Der/die Autor(en) 2018

O. Bendel (Hrsg.), Pflegeroboter, https://doi.org/10.1007/978-3-658-22698-5_9
} 
Versorgungsbedarfs; 2) ein wachsender, zum einen aus benannten demografischen Gründen (steigende Anzahl pflegebedürftiger Menschen) sowie aus Gründen erheblicher beruflicher Rekrutierungsprobleme nicht mehr zureichend $\mathrm{zu}$ deckender Fachkräftebedarf; 3) sich verringernder Anteil der durch Angehörige erbrachten häuslichen Pflege aufgrund wachsender Mobilität des familiären Nachwuchses, insbesondere der zunehmenden Berufstätigkeit von Frauen. Verschiedenen Berechnungen nach wird von einer sich bis zu 300.000 bis 500.000 VZ̈̈ steigernden Pflegepersonallücke im Jahr 2030 ausgegangen (Ehrentraut et al. 2015).

Von daher ist es naheliegend, durch technisch autonome Assistenzsysteme die drohende Personallücke wenigstens zu einem Teil zu schließen. Gemäß dieser Logik wurde im europäischen Raum ein Förderprogramm eingerichtet mit dem Ziel, durch neue assistive Technologien ein möglichst langes selbstständiges Leben im Alter in einer selbst gewählten Umgebung auch bei zunehmender Beeinträchtigung zu gewährleisten. Es sollen funktionelle Verluste bei älteren Menschen in der häuslichen Umgebung ausgeglichen, Alltagskompetenzen aufrechterhalten, darüber hinaus präventive, aber auch rehabilitative Maßnahmen effektiver unterstützt werden. Professionelle Akteure sollen durch systematischen Austausch medizinisch und pflegerisch relevanter Informationen engmaschiger in das System der Gesundheitsversorgung eingebunden werden. Insbesondere Pflegefachkräfte sollen durch technisch autonome Assistenzsysteme im Umkreis der Robotik physisch und kognitiv entlastet sowie ältere Menschen bei der Aufrechterhaltung von Selbstständigkeit und der Ausübung elementarer Tätigkeiten der Selbstversorgung unterstützt werden. Auch wenn die entsprechenden Forschungsund Entwicklungsprogramme primär dem Zweck der gesundheitlichen Versorgungssicherheit im Alter durch moderne Technologien dienen sollen, so sind damit zugleich Förderinteressen jener Hightech-Wirtschaftsbranchen verschränkt, deren Vertreter in die politische Agenda bspw. der EU an prominenter Stelle einbezogen waren, während Interessensorganisationen älterer Menschen oder verschiedener caring professions dagegen eher eine marginale Rolle spielten (Mantovani und Turnheim 2016, 246 f.).

Das effektive Funktionieren unterstützender Technologien ist nicht nur von konstruktiven, sondern auch von soziokulturellen Voraussetzungen abhängig. Dazu gehören insbesondere gesellschaftliche Altersbilder wie bspw. Vorstellungen über mögliche, in den letzten Jahrzehnten tatsächlich immer deutlicher sichtbare Potenziale des Alters, ebenso aber auch Vorstellungen von konkreter, situativ abhängiger Hilfebedürftigkeit. Konstruktiv einzubeziehen sind ebenso differenzierte Erkenntnisse über persönliche Lebenswelten älterer Menschen, ihr häusliches und nachbarschaftliches Wohnumfeld (Peek et al. 2014). Forschungsdesiderata bestehen hinsichtlich empirisch gehaltvoller Erkenntnisse vorhandener kreativer Bewältigungsstrategien des Alltags einerseits sowie wiederkehrender Probleme andererseits (Domínguez-Rué und Nierling 2016). Was erscheint älteren Menschen in Abhängigkeit von konkreten Lebenslagen als technisch hilfreich? Welche konstruktiven Anforderungsanalysen an technische Entwickler ergeben sich daraus (Krings et al. 2014; Simon et al. 2018)? Diese empirischen Aspekte sind nicht unerheblich im Hinblick auf jene Fragen, mit welchen wir uns im Folgenden befassen 
werden; das heißt, mit Fragen der ethischen Bewertung und Beurteilung technisch hochkomplexer Artefakte, deren Einsatz im Umkreis sehr vulnerabler Personen unter bestimmten Bedingungen problematisch sein kann.

\subsection{Ziele und Erwartungen}

Ein Grundsatz bei der Entwicklung und Anwendung verschiedener Assistenzsysteme im Berufsfeld Pflege lautet, dass sie nicht nur der physischen Unterstützung oder dem Ausgleich funktioneller Verluste, sondern auch der Verbesserung von Lebensqualität dienen sollen. Dabei handelt es sich um analytische Bewertungskonstrukte, die von der jeweils eingenommenen Beurteilungsperspektive und den für sie bspw. in institutionellen Kontexten maßgebenden, wissenschaftlich standardisierten Bewertungssystemen abhängig sind. „Lebensqualität“ wird in gesellschaftlichen Untersuchungszusammenhängen bekanntlich soziologisch anders definiert und methodisch gemessen als in Kontexten der Versorgung gesundheitlich eingeschränkter Personen (Kruse 2003).

Die Frage nach der Beurteilungsperspektive stellt sich nicht nur als ein wissenschaftsinternes Problem. Die Frage stellt sich vielmehr auch als eine der politischen Ökonomie, das heißt der Erwartung zahlreicher administrativer Entscheider an technische Entwicklungen, inwieweit sich angesichts des demografischen und epidemiologischen Wandels finanzielle Zusatzbelastungen durch technische Rationalisierungen begrenzen, kurzum durch Technik personelle Einspareffekte erzielen lassen. Damit erhebt sich aber eine grundsätzliche Frage nach der tatsächlichen technischen Substituierbarkeit höchst persönlich zu erbringender Hilfe-, Unterstützungs- oder Beratungsaufgaben bzw. nach dem Grad und dem Umfang ihrer Substituierbarkeit. Diese Erwartungen richten sich auch auf die Einführung robotischer Systeme. Sie sind keineswegs illegitim, schon allein aus zwei Gründen: In bestimmten Bereichen und im Hinblick auf bestimmte (funktionelle) Aufgaben könnte der maschinelle Ersatz (etwa in Bereichen schambesetzter Intimität oder auch der bloß repetitiven Verrichtung fachlich weniger anspruchsvoller unterstützender Tätigkeiten) willkommen sein. In dieser Hinsicht bedarf es klarer Abund Eingrenzungen. Diese hängen aber nicht nur von operationellen Einschätzungen pflegerischer Aufgaben ab, sondern auch von den damit in den meisten Fällen verbundenen emotional bedeutsamen Anteilen persönlicher Zuwendung. Es sind daher differenzierte Beurteilungsmaßstäbe des substituierenden Einsatzes zum Beispiel robotischer Unterstützungssysteme zu entwickeln, abhängig wiederum von einer Vielzahl moralischer Standpunkte und unterschiedlicher ethischer Bewertungsperspektiven.

Es ist im Sinne differenzierter Bedarfsanalysen sowie anspruchsvoller Evaluationen auf eine Klärung der Beurteilungsperspektiven zu drängen. Ob die Perspektive der Betroffenen oder der Angehörigen eingenommen wird oder die Perspektive professioneller Akteure oder die Perspektive der politischen Öffentlichkeit, hat Auswirkungen auf die Bewertung. Im Hinblick auf eine Perspektive der politischen Öffentlichkeit werden zukünftig gesellschaftliche Diskurse zu immer dringlicher werdenden Fragen zu führen 
sein, die da lauten: Wie wollen wir im Alter leben? Wie stellen wir uns gutes Alter vor? Was wird in der Öffentlichkeit unter menschenwürdigem Altern verstanden? ${ }^{1}$ Welche subjektiven und objektiven Anforderungen bestehen hinsichtlich einer guten pflegerischen Versorgung älterer Menschen?

Zur Beantwortung dieser Fragen erscheinen uns v. a. Ansätze einer Ethics of Care, einer Ethik der Sorge oder der Fürsorge, angemessen und zielführend zu sein. Denn sie beruht auf bestimmten anthropologischen Annahmen, die überdies mit charakteristischen Eigenschaften pflegerischen Handelns interferieren. Dabei wird sich auch zeigen, dass Optionen bzw. Wünsche bezüglich bestimmter technischer Hilfen mit - gewiss variierenden - persönlichen Selbstverständnissen ihrer Adressaten korrelieren.

\subsection{Zur ethischen Bewertung autonomer Assistenzsysteme}

\subsubsection{Normativität}

Die Beantwortung vor allem der Frage nach der Erwünschtheit und Angemessenheit technischer Hilfsmittel ${ }^{2}$ erfolgt mehr und mehr im Zusammenhang mit ethisch begründeten, verallgemeinerungsfähigen Bewertungskriterien. Herkömmliche Bewertungskriterien autonomer Assistenzsysteme sind beispielsweise die der Selbstbestimmung, der personalen Integrität (Schutz bzw. freie Entfaltung der Persönlichkeit), der Sicherheit, aber auch Unabhängigkeit, die normative Geltung beanspruchen. $\mathrm{Zu}$ bewerten sind ferner der Erhalt oder die Steigerung von Wohlbefinden. Beim Wohlbefinden handelt es sich, ähnlich wie bei Lebensqualität, um ein zu Zwecken methodisch standardisierter Messverfahren entworfenes wissenschaftliches Konstrukt persönlicher Selbstzuschreibungen auf physischer sowie auf psychoemotionaler Ebene. Die analytischen Ergebnisse von Selbstzuschreibungen sind wiederum abhängig von interpersonalen sowie sozialen Faktoren der gesamten Lebenslage und der Lebensweise einer Person und als solche zu interpretieren.

Angewandte Ethiken zeichnen sich u. a. durch einen konkreten Fallbezug aus. Dabei handelt es sich um die Anwendung der als gültig anerkannten Normen auf einen durch bestimmte empirische (auch kommunikativ erschließbare) Daten beschreibbaren Fall und die Überprüfung, welche der als legitim geltenden Handlungsnormen zur Lösung eines konkreten Entscheidungsproblems unter Berücksichtigung individuell stark variierender persönlicher Wertesysteme am angemessensten sind. Angewandte Ethiken setzen also empirisch präzise Kenntnisse eines Sachverhalts voraus. Deren evaluativer Bedeutsamkeit kann wiederum am besten durch den bereits benannten mehrperspektivischen Ansatz entsprochen werden.

\footnotetext{
${ }^{1}$ Wir verweisen in diesem Zusammenhang auf Arbeiten in Kruse et al. (2012).

${ }^{2}$ Dieser herkömmliche Begriff wird der in autonomen Systemen sich von Grund auf wandelnden Zweck-Mittel-Rationalität nicht mehr gerecht. Vgl. Rammert (2003).
} 


\subsubsection{Anthropologische Bedingungen}

Von welchen anthropologischen Annahmen gehen wir aus - zum einen zur Begründung einer Ethics of Care und der durch sie einzunehmenden Beurteilungsperspektive, zum anderen als konzeptionelle Basis der theoretischen Begründung und empirischen Beschreibung pflegerischen Handelns als eine spezifische Ausprägung von Care? Anthropologische Annahmen verstehen sich als Antworten auf die Frage, was es bedeutet, ein Mensch zu sein (conditio humana). Jenseits eines extrem individualistischen Selbstverständnisses unserer Kultur gilt es sich weiterhin der Tatsache bewusst zu sein, dass sich menschliches Leben unter historisch variierenden Bedingungen physischer Abhängigkeiten und sozialer Angewiesenheiten vollzieht. In ganz elementaren Bereichen ihres Lebens machen Menschen die Erfahrung wechselseitiger Abhängigkeiten, welche sich in sublimer Weise in moralischen Anschauungen manifestiert. Je mehr sich Menschen ihrer gegenseitigen Abhängigkeiten und Angewiesenheiten bewusst werden, umso mehr wird ihnen auch ihre Verletzlichkeit bewusst. Als eine Antwort auf diesen fundamentalen Erfahrungshintergrund kann das Prinzip der Schonung betrachtet werden (Siep 2004). In diesem Zusammenhang lässt sich auch die soziale Bedeutsamkeit eines rein biologischen Faktums herausstellen: Bereits mit seiner Geburt ist der Mensch auf Hilfe, Zuwendung und Anerkennung seiner sozialen Umgebung angewiesen, und er bleibt es in unterschiedlichen Graden in Abhängigkeit von vorhersehbaren und nicht vorhersehbaren Krisen als Wendepunkten persönlicher bzw. gemeinschaftlicher Lebensgeschichte dauerhaft. Diese in anthropologisch tief sitzenden, durch variationsreiche Sozialtechniken gewiss modifizierbaren Grundstrukturen menschlichen Lebens scheinen durch Sichtweisen infrage gestellt zu werden, in welchen individuelle Lösungen für gesellschaftlich erzeugte Probleme im Vordergrund stehen (vgl. Beimborn et al. 2016, insbes. S. 314; van Dyk 2009).

\subsubsection{Spezifika einer Ethics of Care}

Vor dem Hintergrund vorstehend explizierter anthropologischer Tatsachen stehen im Zentrum einer perspektivisch auf Fürsorge ausgerichteten Ethics of Care (vgl. Held 2005; Conradi 2010) Fragen menschlichen Gedeihens, persönlichen Wohlergehens und biografischen Gelingens. Wichtige Impulse für eine Ethics of Care hat bspw. Ludwig Siep (2004) in seiner auf verschiedene Anwendungsszenarien ausgerichteten Konkreten Ethik gegeben. Deren Anspruch besteht nicht nur darin, Antworten darauf zu finden, wie „Voraussetzungen schweren Leidens“ beseitigt werden können. Vielmehr geht es auch darum, indisponible Rechte von Personen zu schützen. Diese Rechte kulminieren gewissermaßen in dem Anspruch, dass Menschen als kooperationsbedürftige und kooperationsfähige Wesen in genau diesem Möglichkeitshorizont gefördert werden. Diesen Zusammenhang akzentuieren besonders Beimborn et al. (2016) in ihrem Entwurf 
eines sozialwissenschaftlich-interdisziplinär fundierten ethischen Bewertungssystems altersbezogener moderner Technologien.

In unseren modernen Gesellschaften sind Wege einer auf Kooperation beruhenden bzw. zielenden Arbeitsteilung zugleich Wege der Förderung jener Bereiche, von denen ein bestandswichtiger Beitrag für das gesellschaftliche Leben erwartet wird. Eine der zentralen Herausforderungen bei der Entwicklung altersbezogener Technologien besteht darin nachzuweisen, inwieweit durch spezielle technische Innovationen ein Beitrag zur Kompensation unterschiedlicher, sozial oder biologisch bedingter Einschränkungen oder Benachteiligungen geleistet werden kann. Diese lediglich auf Kompensationen altersbedingter Defizite eingeschränkte technologische Entwicklungsperspektive muss jedoch erweitert werden, weil andernfalls ein selbst im höheren Alter noch innervierbares Aktivierungs- bzw. Kreativitätspotenzial vernachlässigt würde (Remmers und HülskenGiesler 2012). Die daraus ableitbare normative Prämisse würde also lauten, technische Entwicklungen so auszurichten und Produkte in der Weise zu gestalten, dass auf diesem Wege in concreto Nutzer dazu befähigt werden, an gesellschaftlichen Lebensprozessen teilhaben und, unter der Voraussetzung, dass diese Bedingungen erfüllt sind, ein sinnhaftes, gutes Leben unter ihresgleichen führen zu können.

Bei dieser Argumentation bedienen wir uns eines von Nussbaum (2011) eingeführten theoretischen Ansatzes, welcher unter dem Titel eines Capability-Approaches firmiert (dazu auch Beimborn et al. 2016, S. 320 f.). Diese Engführung zwischen einer Ethics of Care und dem Capability-Ansatz (vgl. Remmers 2010) ist für die Bewertung assistierender Technologien deswegen von besonderer Bedeutung, weil damit eine neue, erweiterte Sicht auf ihre Funktionalität eingenommen wird: nicht mehr die kompensatorische Funktion steht im Vordergrund, mithin das allein auf Hilfe angewiesene Individuum, sondern das Individuum in seiner Potenzialität, in seiner Tendenz der Selbstaktualisierung und in seiner Bildsamkeit. Befähigung als ethisches Bewertungskriterium ist deswegen bedeutsam, weil damit einem von seiner besitzindividualistischen Genese abgekoppelten Autonomieprinzip als Telos, das heißt als ein Ziel der Befähigung, Rechnung getragen wird: Die Befähigung, seinem Leben praktisch einlösbare Ziele setzen zu können (Beimborn et al. 2016, S. 321).

Auf Grundlage der von uns benannten anthropologischen Annahmen (fundamentale, jedoch geschichtlich variierende physische Abhängigkeiten und soziale Angewiesenheiten von Menschen auch als Index ihrer Versehrbarkeit) sowie jener normativen Prämissen einer Ethics of Care (Schutz indisponibler Rechte, Würdigung menschlicher Kooperations- und Gestaltungsfähigkeit) wird ein differenzierter Zugang zu ganz bestimmten Struktureigenschaften pflegerischen Handelns möglich. Da Care stets in einen gesellschaftsgeschichtlich variablen Lebenszusammenhang eingebettet ist, ergeben sich normativ relevante, für Lebenszusammenhänge in ihrer strukturellen Wechselseitigkeit höchst charakteristische, aber vielseitige Verantwortungs- und Anerkennungsverhältnisse. Als würdevoll gilt menschliches Leben unter Bedingungen gegenseitiger Anerkennung, die auch durch Asymmetrien in der Beziehungsform (zum Beispiel bei unterschiedlich verteilten Kompetenzen) nicht durchkreuzt werden. Durch diese Grundtatsachen ist auch Pflege charakterisiert, die im Kern eine Beziehungsarbeit darstellt. 


\subsubsection{Zwischenbemerkung: Einige Charakteristika pflegerischen Handelns}

Pflege ist eine Dienstleistung professioneller Hilfe für Menschen, die bedingt durch Erkrankungen, Behinderungen oder Abbauprozesse Einschränkungen erleben, welche die bio-psycho-soziale Integrität dieser Menschen zutiefst berühren können. In dem Maße, wie sich solche Leidenszustände krisenhaft zuspitzen, treten Bedürfnisse nach Sicherheit und erfolgreicher Bewältigung von Spannungs- und Stresssituationen in den Vordergrund. Auf elementarer Ebene kann daher Pflege als eine an den Grundbedürfnissen hilfebedürftiger Menschen ansetzende Beziehungsarbeit charakterisiert werden, welche neben wissenschaftlich-fachlichen Anforderungen ebenso spezifisch emotionalen Anforderungen zu genügen hat (Strauss et al. 1980). In dieser Hinsicht besteht eine gewisse Affinität zur meist weiblich zugewiesenen Haus- und Familienarbeit.

Der direkte Umgang mit körperlich stark eingeschränkten Menschen stellt in der Regel eine hohe physische Belastung des Pflegepersonals dar. Die Naturgebundenheit pflegerischen Handelns kommt aber auch in einer anderen Tatsache zum Ausdruck. Pflege als Beziehungsarbeit vollzieht sich im psychophysischen Medium leiblicher Gegenseitigkeit, die eine gewisse Diffusität sozialer Rollen mit sich bringt mit dem Risiko einer Symbiose ohne Schutz vor grenzenloser Verausgabung (Stichwort: Burnout) sowie der Gefahr des Missbrauchs eigener Machtpositionen.

Weitere Schwierigkeiten resultieren aus einem sehr anspruchsvollen Synchronisationsbedarf von Tätigkeiten wie: Organisation von Versorgung, persönliche, stets leiblich präsente Zuwendung sowie affektive Balance. Die Tatsache, dass pflegerische Tätigkeiten an natürlichen, zyklisch wiederkehrenden, jedoch situativ variierenden (Grund-)Bedürfnissen auszurichten sind, macht sie zeitlich nur schwer planbar und kaum kontrollierbar (Weishaupt 2006; Dunkel und Weihrich 2010). Massiv wird zudem das Erfolgserleben dadurch erschwert, dass - im Gegensatz bspw. zur Herstellung materieller Güter oder der Bearbeitung von Verwaltungsvorgängen - Arbeitsresultate sich rasch verflüchtigen. Vor allem bei therapeutischer Aussichtslosigkeit, Siechtum und Verfall fehlt ihnen eine materielle Anschaulichkeit des Erfolgs, der überdies anders zu bemessen ist (Belan und Schiller 2016).

Diese phänomenologisch weitaus differenzierter darstellbaren Formen und Eigenschaften pflegerischer Arbeit betrachten wir als einen mehrere Wahrnehmungsschichten gleichzeitig affizierenden Kernbereich der Pflege. Ihres zyklischen Charakters wegen rangiert sie als „Beziehungsarbeit“ in der Statushierarchie therapeutischer Berufe eher niedrig. Bedacht werden sollte auch, dass methodische Verfahrensweisen der Wirksamkeitsprüfung pflegerischer Maßnahmen auf die Notwendigkeit einer (experimentellen) Isolierung einzelner Faktoren angewiesen, angesichts der Komplexität und Diffusität pflegerischer Handlungen aber häufig zum Scheitern verurteilt sind. Hinzu kommt schließlich, dass das im praktischen Umgang mit Hilfebedürftigen erworbene Wissen eine genuine, hochwertige, streng genommen aber „vorrationale“, qua mimetischem Vermögen konstituierte Wissensform darstellt, welche wissenschaftlich zunächst einmal 
kaum anschlussfähig zu sein scheint, freilich durch analytisch hoch differenzierte Verfahren erschließbar ist.

Mit der Verwissenschaftlichung und Technisierung des Medizinsystems sowie der Pflege in einem zweiten großen Modernisierungsschub um die Mitte des 20. Jahrhunderts setzen sich Trends durch, die - neben dem Erzielen besserer Krankenbehandlungsergebnisse - durch den Einsatz elektronischer Datenverarbeitungssysteme für eine Rationalisierung klinischer Arbeitsabläufe mit kostenökonomischen Einspareffekten sorgen. Mit solchen Trends der Formalisierung und Standardisierung prallen aber zwei inkommensurable Logiken aufeinander: Die eigensinnige, in die soziale Lebenswelt von Personen verflochtene Logik professioneller Beziehungsarbeit (in Pflege, teilweise auch in hausärztlicher Medizin) einerseits sowie die in technologische Programme übersetzten Logiken von Ökonomie und planender Verwaltung andererseits (Glaser et al. 2008; vgl. ebenso Remmers 2015). Zweifellos kann mit dynamisch gesteigerten Technologieentwicklungen eine zunehmende Effektivität bspw. auf instrumenteller Ebene medizinischer und zumeist nachgeordneter pflegerischer Eingriffe und ein damit verbundener Zuwachs vitaler Sicherheit erzielt werden. $\mathrm{Zu}$ verzeichnen sind allerdings auch Widerstände, die sich gegen technisch induzierte Entfremdungsphänomene (z. B. Verlust der persönlichen, körperlich-leiblichen Nähe zum Patienten) und Deprofessionalisierungseffekte richten (Darbyshire 2004; Remmers und Hülsken-Giesler 2011; Nagel und Remmers 2012).

Für Grunwald (2013) beruht das Ideal von Technik, man könnte auch sagen: ihr funktionelles Apriori, darauf, in möglichst hohem Maße dem konstruktiven Prinzip situationsinvarianter Regelhaftigkeit zu genügen. Ein maximaler Grad der Regelhaftigkeit technischer Verfahren und der dadurch ermöglichten Reproduzierbarkeit identischer Ergebnisse kann nur durch einen möglichst hohen Grad der Kontextunabhängigkeit erzielt werden. Die praktische Abstraktion von situativen Besonderheiten ist eine Voraussetzung regelhaften Funktionierens, die ihrerseits von sozialer Bedeutung ist. Handlungstheoretisch gewendet, kann nämlich durch technische Schematisierung von Verfahren bzw. durch Berechenbarkeit maschineller Funktionsabläufe Verlässlichkeit gestiftet werden, welche mit Wünschen nach Erwartungssicherheit korreliert. Sie entlastet soziale Akteure vom Improvisationsaufwand ständig neuer Situationsdefinitionen und der Begründung von Handlungsoptionen. Gewiss, durch eine Technifizierung von Handlungskontexten werden, wie Grunwald (2013) betont, immer auch Freiheit und Individualität bedroht. In diesem Zusammenhang lassen sich auch die Skepsis und vielfach zu beobachtenden Vorbehalte gegenüber Technik aufseiten der Pflegekräfte erklären: Sie verdanken sich einer sicheren Intuition, dass dem Prinzip situationsinvarianter Regelhaftigkeit gehorchende technische Artefakte oder Verfahren sich in professionelle, das heißt auch gemäß den Prinzipien der Individualisierung und Kontextualisierung geordnete Handlungszusammenhänge nur bedingt, das heißt unter Beachtung bestimmter Kautelen, integrieren lassen.

In Bezug auf die hohen körperlichen Belastungen kann der Einsatz arbeitserleichternder maschineller Unterstützungstechnologien als segensreich gewertet werden. Es stellt sich darüber hinaus aber die Frage, in welchen Anteilen und Ausmaßen 
Beziehungsarbeit durch freundliche Companions ersetzt werden kann und darf. Dies ist nicht nur eine ethische, sondern auch eine arbeitssoziologische und -psychologische Frage angesichts mindestens dreier charakteristischer Phänomene: der zyklischen Organisationsform von Pflege, bestimmter affektueller Charakteristika der Tätigkeit sowie jener phänomenalen Besonderheiten einer leiblichen Responsivität (Waldenfels 1994): einer Gegenseitigkeit des Fühlens und Gefühltwerdens, des Berührens und Berührtwerdens - was im Übrigen von hohem therapeutischen Wert ist. Die Einbeziehung von Assistenzsystemen in pflegerische Arbeitsprozesse wird sich als produktiv oder entlastend nur dann erweisen, wenn dadurch jene originären, in einer leiblichen Intentionalität fundierten multisensorischen Wahrnehmungsfähigkeiten nicht nur nicht angetastet, sondern einen größeren Entfaltungsspielraum erhalten werden (vgl. Oleson 2006). Diese phänomenalen Besonderheiten sind u. a. der Grund jener Ambivalenzen von Pflegefachkräften bei der Bewertung des Einsatzes von Überwachungstechnologien bei pflegebedürftigen Personen (Pols 2012).

\subsubsection{Zum Einsatz autonomer Assistenzsysteme in der Pflege}

Bezüglich des Einsatzes von Robotern möchten wir zunächst eine provisorische Unterscheidung vornehmen zwischen Assistenzrobotern, Therapierobotern und Interaktionsrobotern. $\mathrm{Zu}$ den Assistenzrobotern werden im Allgemeinen diejenigen gerechnet, welche die Medikation unterstützen (zeitlich sicher programmierte Abgabe fest programmierter Dosen von Medikamenten). Ferner der als Care-O-bot 4 entwickelte Roboter, der manuelle Tätigkeiten (Greifen, Anreichen) ausführt und z. B. das Anreichen von Speisen und Getränken auf Kommando ausführt. Schließlich ein intelligenter Pflegewagen, der auf Anforderung herbeirollt, Pflegeutensilien vorrätig hält und ihren Verbrauch dokumentiert, um stets vollständig bestückt und einsatzbereit zu sein. Zu den Assistenzrobotern gehören auch die zur physischen Alltagsunterstützung in der häuslichen und stationären Pflege wie Heben und Tragen eines Patienten entwickelten Systeme. Unter diese Kategorie fallen ebenso Exo- oder Außenskelette, also Mensch-Maschine-Systeme, welche menschliche Intelligenz mit maschineller Kraft kombinieren, indem sie die Bewegungen des Trägers unterstützen oder verstärken.

Exoskelette sollen zukünftig vor allem dort zum Einsatz kommen, wo menschliche Arbeit nicht sinnvoll durch Automatisierung oder Robotik-Systeme ersetzt werden kann. Hierzu zählen bestimmte Arbeitsprozesse in der industriellen Produktion, beispielsweise in der Automobilbranche, aber auch körperlich schwere Arbeiten im Baugewerbe, der Logistik oder im Pflegebereich. Schließlich können den Assistenzrobotern auch bestimmte Tracking-Systeme zugerechnet werden, deren Einsatz vor allem der örtlichen Selbst- oder Fremdkontrolle kognitiv eingeschränkter, möglicherweise an einer leichten Demenz leidender Personen dient.

$\mathrm{Zu}$ den Therapierobotern gehören zum Beispiel diejenigen, die die Mobilität unterstützen. $\mathrm{Zu}$ denken ist hier beispielsweise an einen im Projekt ROREAS entwickelten 
„Roboter-Companion“, der Patienten beim Gangtraining in der Klinik und bei häuslichen Übungen automatisch unterstützt. Er dient ebenso als Erinnerungshilfe oder Navigationshilfe. $\mathrm{Zu}$ den wahrscheinlich bekanntesten Therapierobotern gehört der speziell für Demenzkranke entwickelte Emotionsroboter PARO. Er ist einer Robbe nachgebildet und reagiert auf taktile und verbale Ansprache mit eigenen Bewegungen (Augenlider, Extremitäten, Heben und Senken des Kopfes) und mit Lautbildungen. Mit PARO sollen seelisches Wohlbefinden erzeugt, Stress reduziert und Einsamkeitsgefühle gelindert werden.

Im Bereich der Interaktionsroboter gibt es viele Entwicklungsprojekte, in denen an Prototypen zu sozialen Interaktionsfähigkeiten geforscht wird. $\mathrm{Zu}$ nennen ist hier bspw. die ALIAS-Roboterplattform, welche drei Kernfunktionen beinhaltet: Die erste Funktion bezieht sich auf die Kommunikation. Ziel ist es, die Unterhaltung mit Partnern zu unterstützen und Kontakte aufrechtzuerhalten. Dies erfolgt durch Nutzung von modernen Kommunikationstechnologien und von Ressourcen des Web 2.0, die einen Zugriff auf soziale Netzwerke ermöglichen sollen. Die zweite Funktion bezieht sich auf die Aktivität: Der Roboter bietet Möglichkeiten physischer und kognitiver Aktivitäten. Zu diesem Zweck steht eine Vielfalt an Spielen zur Verfügung, die auf dem Display genutzt werden können. Falls eine Person diese Funktionen in nicht ausreichendem Maße selbstständig nutzen kann, wird die Roboterplattform proaktiv deren Nutzung anregen. Die dritte Funktion ist die der Assistenz. Diese Funktion kann an verschiedene Nutzergruppen mit unterschiedlichen Unterstützungsbedarfen adaptiert werden. Beispielsweise erinnert die Roboterplattform Nutzerinnen und Nutzer an fällige Medikamenteneinnahmen oder an geplante Termine. Über einen Fernzugriff können Angehörige diese Funktionen steuern bzw. selbst nutzen.

\subsubsection{Ethische Beurteilung der Folgen des Einsatzes autonomer Assistenzsysteme}

Von andernorts erörterten Akzeptanzfragen (Remmers und Hülsken-Giesler 2011) sind normativ-ethisch übergreifende Beurteilungen technischer Entwicklungen und Artefakte zu unterscheiden (Grunwald 2005). In einer zumeist konsequenzialistischen, das heißt an den Folgen von Handlungen, Interventionen oder auch technischen Installationen ausgerichteten Beurteilungsperspektive kommt es häufig dazu, dass verschiedene, aus unterschiedlichen Sichtweisen als jeweils angemessen geltende Bewertungskriterien wie bspw. Selbstbestimmung und Wohlbefinden kollidieren. $\mathrm{Zu}$ solchen Bewertungsdiskrepanzen kann es auch bei der Beurteilung des konkreten Einsatzes autonomer Assistenzsysteme kommen. In einem bestimmten Falle kann zum Beispiel durch den Einsatz assistierender Technologien häusliche Unabhängigkeit erzielt werden. Dies mag ein Wert an sich sein, der aber mit persönlichem Wohlbefinden nur beschränkt kongruent ist. Es könnte nämlich sein, dass technisch unterstützte häusliche Unabhängigkeit zu einem Sicherheitsgefühl (vor allem auch bei betreuenden Angehörigen) führt, während damit gleichzeitig eine Verminderung sozialer Kontakte und vermehrte Einsamkeit verbunden ist (so auch 
Kamphof 2016, S. 176). In diesem Falle wäre zu klären, inwieweit ethische Kriterien wie Unabhängigkeit oder Sicherheit durch andere ethische Kriterien wie Versorgungsqualität oder persönliche Zuwendung übertrumpft werden. Aus der Perspektive einer Care-Ethik würde das Konzept personaler Autonomie grundsätzlich nicht in Zweifel gezogen. Es würde jedoch, im Anschluss an Sandel (1998), auf damit verbundene Einseitigkeiten verwiesen werden, das Leben als eine „Robinsonade“ zu begreifen. Stattdessen würde das Konzept einer relationalen Autonomie (vgl. etwa Simon und Nauck 2013) favorisiert mit dem Hinweis darauf, technische Arrangements so zu gestalten, dass bspw. Vereinsamung möglichst ausgeschlossen werden kann.

Ein gewichtiger Einwand hinsichtlich des Einsatzes autonomer Assistenzsysteme lautet, dass sie letztlich als Mittel der Rationalisierung eingesetzt werden. ${ }^{3}$ Unter ethischen Gesichtspunkten z. B. der Steigerung von Wohlbefinden wären solche Rationalisierungen gerechtfertigt und sogar erwünscht, wenn damit beispielsweise größere Zeitreserven des Pflegepersonals für besonders versorgungsbedürftige ältere Menschen oder Patienten gewonnen werden könnten. Auch die durch den Einsatz von Servicerobotern (Heben, Lagern) zu erzielenden physischen Entlastungen sind erwünscht. Ethisch relevante Probleme ergeben sich dann, wenn Pflegebeziehungen in ganz elementaren Bereichen (körperliche Nähe, subtile, differenzierte Wahrnehmung, therapeutisch bedeutsame Berührung) technisch substituiert werden sollen:

(...) It clearly can be argued that peaceful, even loving, interaction among humans is a moral good in itself. (...), we should probably distrust the motives of those who wish to introduce technology in a way that tends to substitute for interaction between humans. (...) for a social mammal such as a human, companionship and social interaction are of crucial psychological importance (Whitby 2012, S. 238).

Neueren Studien zufolge können telematische Assistenzsysteme zu einer Reduzierung menschlicher Kontakte führen und damit zu einer sozialen Isolation älterer Menschen (Sharkey und Sharkey 2012a; Sparrow und Sparrow 2006; Sparrow 2002). Familienmitglieder fühlen sich von gewissen Verpflichtungen entlastet, was gewiss auch als ein legitimer Wunsch gelten kann (Sharkey und Sharkey 2012b). Dadurch hervorgerufene Vereinzelungen machen auf der anderen Seite wiederum stärkere Kontrollen notwendig (Sorell und Draper 2014). Durch den Einsatz bspw. von AAL-Technologien muss soziale Isolation zwangsläufig nicht ansteigen. Immer kommt es auf die Einzelfallbeurteilung an (vgl. Sorell und Draper 2012; Murray et al. 2011; Pols 2010).

Auch ist in jedem Einzelfall zu prüfen, ob durch eine Substitution menschlicher Hilfe durch das technische Assistenzsystem der moralisch (teils rechtlich) begründete Anspruch eines jeden Menschen auf Anerkennung und Zuwendung verletzt wird (vgl. etwa Borenstein und Pearson 2012). Zumindest bei telematischer Überwachung werden Tendenzen einer Ent-Individualisierung beklagt (Sävenstedt et al. 2006).

\footnotetext{
${ }^{3} \mathrm{Im}$ Folgenden stützen wir uns auf ein unlängst verabschiedetes Gutachten: Hülsken-Giesler und Remmers (2017).
} 
Ernst zu nehmen sind kritische Hinweise, dass mit der Einführung von Assistenztechnologien ein Einstellungswandel verbunden sein kann. Dies betrifft zum einen bestimmte Erwartungen älterer Personen gegenüber ihren Mitmenschen, zum anderen gesellschaftliche Vorstellungen von Verantwortung (vgl. Hinman 2009; Sparrow und Sparrow 2006). Bei der Diskussion dieser ethischen Bedenken sollten allerdings der seit etlichen Jahrzehnten sich vollziehende Wandel von Familienstrukturen und die damit zusammenhängenden Veränderungen affektiver Bindungen sowie subjektiv zugeschriebener Verantwortlichkeiten erwachsener Kinder gegenüber ihren Eltern bedacht werden.

Bei der ethischen Bewertung, welche Folgen der Einsatz von Robotern für soziale Interaktionen älterer Menschen hat, sollten Studien von Berkman und Syme (1979) zur Kenntnis genommen werden. Ihre Ergebnisse besagen, dass Personen, denen soziale Beziehungen fehlen, ein höheres Risiko vorzeitigen Versterbens haben. Auch sind wenige soziale Beziehungen, seltene Teilnahme an sozialen Aktivitäten und sozialer Rückzug Risikofaktoren für die Abnahme kognitiver Fähigkeiten älterer Personen (Zunzunegui et al. 2003). Demgegenüber gibt es Hinweise darauf, dass soziale Interaktion das Risiko, an Demenz zu erkranken, reduziert (Saczynski et al. 2006).

Aus der Perspektive eines ethischen Konsequenzialismus sowie vor dem Hintergrund einer Ethics of Care lässt sich sagen, dass der Einsatz assistiver Technologien bis hin zu Robotern daran zu bemessen ist, inwieweit soziale Beziehungen, die erwiesenermaßen zur Steigerung subjektiven Wohlbefindens beitragen, gestärkt oder geschwächt werden (Palm 2014; Fine und Spencer 2009; Szebehely und Trydegard 2012). Darüber hinaus ist der intrinsische Wert sozialer Interaktionen zu würdigen:

Social networks are said to imply (1) appreciation, recognition and a feeling of belonging,

(2) intimacy and friendship and (3) emotional and practical support (...) (Palm 2014, mit Verweis auf Fine und Spencer 2009).

Schließlich ist mit Blick auf ethisch bedeutsame Implikationen des Capability Approach $\mathrm{zu}$ fragen, inwieweit gegenwärtige Entwicklungen autonomer Assistenztechnologien eine altersgerechte Unterstützung sozialer Vernetzungsprozesse erwarten lassen, und zwar als Grundlage für soziales Lernen, welches zugleich der Entfaltung kreativer Potenziale zu dienen vermag (Remmers und Hülsken-Giesler 2012).

\subsubsection{Besonderheiten bei Demenzpatienten}

Bei ethischen Fragen des Einsatzes von Therapierobotern (Beispiel: PARO-Robbe) in der Pflege demenziell Erkrankter liegen einer Ethics of Care verschwisterte Perspektiven der Advokation nahe. Nun gibt es überzeugende empirische Studien, die zeigen, dass Demenzkranke auch im fortgeschrittenen Stadium der Erkrankung über Fähigkeiten verfügen, sich emotional mitzuteilen, Wünsche und Bedürfnisse ebenso wie Aversionen und Ablehnungen auf nonverbalem Wege (Blick, Gestik, Mimik) zu signalisieren (Becker et al. 2010a, b). Erfahrene Pflegekräfte wiederum verfügen über ein multisensorisches 
„Deutungsrepertoire“, das heißt über explorative Verfahren wie bspw. das der faszialen Ausdrucksanalyse, durch welches biografisch essenzielle Anliegen dieser Menschen erschlossen werden können. Inwieweit dieses in einer leiblich-sozialen Existenzweise des Menschen (u. a. Becker 2003) verankerte intuitive Vermögen auf epistemischer Ebene künstlicher Intelligenz in robotischen Systemen ersetzt werden kann, ist eine offene Frage.

Wie verhält es sich mit „Lebensqualität“ als ethischem Bewertungskriterium nicht nur von pflegerischen Interventionen, sondern auch von installierten technischen Artefakten? Gemäß Lawton et al. (1996) beinhaltet das Lebensqualitätsmodell bei Demenzkranken folgende Bewertungsdimensionen: a) räumliche Umwelt, b) soziale Umwelt, c) Betreuungsqualität, d) subjektives Erleben und emotionale Befindlichkeit. In dieser vierten Dimension sind Ansatzpunkte für einen legitimen Einsatz von Emotionsrobotern zu prüfen. Sie treten gewissermaßen in eine Konkurrenz mit originär pflegerischen Interventionen. Dazu gehören: auf verbalsprachlicher Ebene gezielte Ansprache und Kommunikation; auf nonverbaler Ebene visuelle, auditive und gustatorische Anregungen zur Förderung subjektiver Erlebnisfähigkeit und zur Vermeidung von Einsamkeit und Isolation. Ob hier von einer echten Konkurrenz gesprochen werden kann, könnte allein schon aus technologischen Gründen problematisiert werden.

Von welchen Voraussetzungen ist beim Einsatz von Emotionsrobotern bei demenziell Erkrankten auszugehen? Ihren algorithmischen Basisfunktionen nach sind Emotionsroboter wie PARO auf reproduzierbare Verhaltensschemata orientiert und programmiert. Es ist ihnen nicht möglich, durch situative Bewertungen eine differenzierte Antwort im Sinne eines auf die persönlichen Gefühlslagen ausgerichteten Verhaltensangebots zu generieren. Sie sind nach rein behavioristischen Mustern und Oberflächenphänomenen menschlichen Orientierungs- und Aktionsvermögens konstruiert. Dies mag sich im Zuge eines zukünftigen Zuwachses an Lernfähigkeit künstlicher Intelligenzen, d. h. der kontinuierlichen Anpassung z. B. robotischer Systeme an ihre Umgebungen, ändern. ${ }^{4}$

Abgesehen von diesen aus einer technischen Bewertungsperspektive zu erörternden Fragen ergeben sich weitere Fragen aus einer empirisch-sozialwissenschaftlichen Perspektive. Zwar gibt es - als Messergebnis auf rein behavioraler Ebene und mit methodischen Einschränkungen (häufig sehr kleine Fallzahlen) - Hinweise auf vermehrtes kommunikatives Verhalten, Entspannung, verminderte Einsamkeitsgefühle, vermehrte neuronale Aktivität, verminderten Stress beim Einsatz von PARO bei Betroffenen sowie auf Entlastungseffekte beim Pflegepersonal (Robinson et al. 2015; Sung et al. 2015;

\footnotetext{
${ }^{4}$ Hochbedeutsam wäre dies, wenn PARO durch einen humanoiden Roboter ersetzt würde. Die Lernalgorithmen solcher Roboter würden verlangen, ,dass Menschen mit ihm , wie mit Menschen " umgehen“ (Decker 2010, S. 51). Das wäre im Falle von Demenzkranken deswegen aber verheerend, weil damit alle Maßstäbe eines professionellen Umgangs verletzt würden, welcher auf Fähigkeiten der Reflexion und der dadurch erst möglichen situativen Abstimmung des Verhaltens beruht.
} 
Wada et al. 2008; Saito et al. 2003). Diese Effekte sind jedoch zeitlich oft sehr begrenzt (Baisch et al. 2018). Im Vergleich des Einsatzes von PARO mit dem des humanoiden Systems NAO und dem eines lebenden Hundes konnten durch PARO und NAO positive Einflüsse auf apathisches Verhalten von Probanden erzielt werden, wobei die kognitive Leistungsfähigkeit der Probanden aus dem NAO-Experiment signifikant absank (Valenti Soler et al. 2015).

Geht man davon aus, dass demenziell Erkrankte auf elementare Formen emotionaler, leiblicher Zuwendung angewiesen sind, so erheben sich prinzipielle ethische Einwände hinsichtlich der artifiziellen Substitution auf struktureller Ebene. Bei PARO wird leibliche Präsenz nur mehr vorgetäuscht, sie bleibt auf reaktive Oberflächenphänomene beschränkt. Analogien mit dem Gebrauch von Stofftieren bei Kindern sind psychologisch deswegen völlig abwegig, weil sie für die kognitiv-emotionale Entwicklung des Kindes wichtige „transitional objects“ (Winnicott 1953) darstellen mit einer spezifischen Intentionalität (als erste fantasiereiche Akte der Kreativität). Der künstlichen, persönlich indifferenten Erzeugung sozial erwünschter Gefühlszustände wohnt insofern ein Täuschungscharakter inne, der als eine Entwürdigung bewertet werden kann. Damit ergeben sich ethische Fragen von Wahrheit und Wahrhaftigkeit, die mit dem Hinweis darauf, dass Hilfeempfänger und helfende Person in zwei verschiedenen Welten des Bewusstseins leben, nicht entkräftet werden können (Hertogh et al. 2004; vgl. auch Remmers 2016). Stattdessen sollte bedacht werden, dass Pflegefachkräfte in ihrer Unterstützung und Hilfe bei Menschen mit kognitiven Einbußen mit moralischen Dilemmata konfrontiert sein können. Es empfiehlt sich deshalb, den Einsatz autonomer Assistenztechnologien wie in diesem Falle der PARO-Robbe möglichst zu begrenzen. Dies umso mehr, als ein zeitlicher Entlastungseffekt für Pflegefachkräfte kaum zu erkennen ist (Baisch et al. 2018). Nicht von der Hand zu weisen sind Gefahren, dass Roboter ein menschlich fürsorgliches Umfeld in beträchtlichem Umfang ersetzen.

\section{$9.4 \quad$ Fazit}

Die gegenwärtige Diskussion zeigt, dass ethische Bewertungen autonomer Assistenzsysteme in der Pflege abhängig sind von den jeweils eingenommenen Beurteilungsperspektiven. Auch wenn normative Ansprüche im Wesentlichen die Respektierung personaler Selbstbestimmung sowie individuell artikulierten Wohlbefindens verlangen, so zeigt die Anwendung dieser Prinzipien, dass vor allem bei inkongruenten Perspektiven widersprüchliche Deutungen einer als entscheidungsbedürftig empfundenen Situation in den Vordergrund treten. Der Umgang mit dabei auftretenden Ambivalenzen ist ohnehin ein Charakteristikum pflegerischen (wie auch ärztlichen) Handelns in Ungewissheit. Genau dieser Tatbestand verlangt ein hohes Maß ethischer Sensibilität bei der Beurteilung technischer Systeme, denen der Anspruch der Erleichterung pflegerischen Handelns bzw. der Verbesserung der Lebensqualität pflegebedürftiger Menschen quasi funktional eingeschrieben ist. Fragen, was der Einsatz robotischer Systeme „mit Menschen 
macht“, denen er auf verschiedenen Ebenen des Daseinsvollzugs zugute kommen soll, sind ebenso wenig von der Hand $\mathrm{zu}$ weisen wie Fragen, mit welchem Recht Gefühlszustände stark vulnerabler Patienten bzw. Bewohner künstlich manipuliert werden dürfen, einzig um einen ohnehin flüchtigen Beruhigungszustand zu erreichen. In welchem Ausmaße dürfen überhaupt robotische Systeme die persönliche Präsenz und lebendige Interaktion von Pflegefachkräften substituieren? Wenn die persönliche Begegnung mit Menschen in Bedrängnis eine elementare Voraussetzung professionellen Handelns ist (wodurch Ansätze von Telehealth oder Telenursing auf ihren menschengemäßen Radius zurückgeschraubt würden), so stellt sich die bis dato viel zu wenig beachtete Frage, unter welchen Voraussetzungen der technischen Entwicklung robotischer Systeme genau diese Sphäre zwischenmenschlicher Begegnung praktisch aufgewertet und verbessert werden könnte (Demand-Pull-Orientierung). Die kontinuierliche Einbindung von möglichen Leistungsempfängern (einschließlich pflegenden Angehörigen) sowie professionellen Akteuren in die Technikentwicklung ist allein schon aus ethischen Gründen geboten.

\section{Literatur}

Baisch, S., Kolling, T., Rühl, S., Klein, B., Pantel, J., Oswald, F., \& Knopf, M. (2018). Emotionale Roboter im Pflegekontext. Empirische Analyse des bisherigen Einsatzes und der Wirkungen von Paro und Pleo. Zeitschrift für Gerontologie und Geriatrie, 51, 16-24.

Becker, B. (2003). Zwischen Autonomie und Heteronomie. Zur Schwellensituation leiblicher Individualität. In T. Christaller \& J. Wehner (Hrsg.), Autonome Maschinen (S. 56-68). Wiesbaden: Westdeutscher Verlag.

Becker, G., Kaspar, R., \& Kruse, A. (2010a). Heidelberger Instrument zur Erfassung der Lebensqualität demenzkranker Menschen (H.I.L.DE). Bern.

Becker, G., Kaspar, R., \& Kruse, A. (2010b). Heidelberger Instrument zur Erfassung der Lebensqualität demenzkranker Menschen (H.I.L.DE) - das Instrument in seinen konzeptionellen Grundlagen und in seiner praktischen Anwendung. In A. Kruse (Hrsg.), Lebensqualität bei Demenz? Zum gesellschaftlichen und individuellen Umgang mit einer Grenzsituation im Alter (S. 137-156). Heidelberg.

Beimborn, M., Kadi, S., Köberer, N., Mühleck, M., \& Spindler, M. (2016). Focusing on the human: Interdisciplinary reflections on ageing and technology. In E. Domínguez-Rué \& L. Nierling (Hrsg.), Ageing and technology: Perspectives from the social sciences (S. 311-333). Transcript.

Belan, D., \& Schiller, C. (2016). Helfen ohne zu heilen: Berufsbedingte Belastungen und Bewältigungsstrategien von Palliativpflegekräften. Bd. 23 der Schriftenreihe Arbeit und Leben im Umbruch. München: Hampp.

Berkman, L. F., \& Syme, L. F. (1979). Social networks, host resistence, and mortality: A nineyear follow-up study of Alameda country residents. American Journal of Epidemiology, 109(2), 186-204.

Borenstein, J., \& Pearson, Y. (2012). Robot caregivers: Ethical issues across the human lifespan. In P. Lin, K. Abney, \& G. A. Bekey (Hrsg.), Robot ethics. The ethical and social implications of robotics (S. 251-265). Cambridge.

Conradi, E. (2010). Ethik und Politik. Wie eine Ethik der Achtsamkeit mit politischer Verantwortung verbunden werden kann. In R. Hartmut \& K. Helen (Hrsg.), Bioethics, Care and 
Gender - Herausforderungen für Medizin, Pflege und Politik. Bd. 4 der Schriftenreihe: Pflegewissenschaft und Pflegebildung. Vandenhoek \& Ruprecht unipress, Universitätsverlag Osnabrück (S. 91-117). Göttingen.

Darbyshire, P. (2004). 'Rage against the machine?': Nurses' and midwives' experiences of using computerized patient information systems for clinical information. Journal of Clinical Nursing, $13,17-25$.

Decker, M. (2010). Ein Abbild des Menschen: Humanoide Roboter. In M. Bölker (Hrsg.), Information und Menschenbild (S. 41-62). Berlin: Springer.

Domínguez-Rué, E., \& Nierling, L. (Hrsg.). (2016). Ageing and technology: Perspectives from the social sciences. transcript.

Dunkel, W., \& Weihrich, M. (2010). Arbeit als Interaktion. In F. Böhle, G. G. Voß, \& G. Wachtler (Hrsg.), Handbuch Arbeitssoziologie (S. 177-200). Wiesbaden.

Ehrentraut, O., Hackmann, T., Krämer, L., \& Schmutz, S. (2015). Zukunft der Pflegepolitik-Perspektiven, Handlungsoptionen und Politikempfehlungen. Friedrich-Ebert-Stiftung (Hrsg.) Bonn. http://library.fes.de/pdf-files/wiso/12140.pdf. Zugegriffen: 23. Mai 2016.

Fine, M., \& Spencer, R. (2009). Social isolation development of an assessment tool for HACC services. A literature review conducted for the New South Wales Department of Disability, Ageing and Home Care (DADHC).

Glaser, J., Lampert, B., \& Weigl, M. (2008). Arbeit in der stationären Altenpflege - Analyse und Förderung von Arbeitsbedingungen, Interaktion, Gesundheit und Qualität. Dortmund.

Grunwald, A. (2005). Zur Rolle von Akzeptanz und Akzeptabilität von Technik bei der Bewältigung von Technikkonflikten. Technikfolgenabschätzung - Theorie und Praxis, 14(3), 54-60.

Grunwald, A. (2013). Technik. In A. Grunwald (Hrsg.), Handbuch Technikethik, Metzler'sche Verlagsbuchhandlung. (S. 13-17). Stuttgart.

Held, V. (2005). The ethics of care. Personal, political and global. Oxford University Press.

Hertogh, C. M. P. M., Mei The, B. A., Miesen, B. M. L., \& Eefsting, J. A. (2004). Truth telling and truthfulness in the care for patients with advanced dementia: An ethnographic study in Dutch nursing homes. Social Science \& Medicine, 59(8), 1685-1693.

Hinman, L. (2009). Robotic companions: Some ethical questions to consider. http://ethicsmatters. net/Presentations/Popular/ICRA2009/Hinman,\%20Robotic\%20Companions--Ethical\%20Considerations.pdf.

Hülsken-Giesler, M., \& Remmers, H. (2017). Autonome Assistenzsysteme in der Pflege: Potenziale und Grenzen aus pflegewissenschaftlicher Sicht (PflegeRobot). Gutachten für den Deutschen Bundestag - vorgelegt dem Büro für Technikfolgen-Abschätzung beim Deutschen Bundestag (TAB).

Kamphof, I. (2016). Seeing again. Dementia, personhood and technology. In E. Domínguez-Rué \& L. Nierling (Hrsg.), Ageing and technology: Perspectives from the social sciences (S. 163-181). transcript.

Krings, B. J., Böhle, K., Decker, M., Nierling, L., \& Schneider, C. (2014). Serviceroboter in Pflegearrangements. Zukünftige Themen der Innovations-und Technikanalyse: Lessons learned und ausgewählte Ergebnisse (S. 63-121). Karlsruhe: KIT Scientific Publishing.

Kruse, A. (2003). Lebensqualität im Alter. Befunde und Interventionsansätze. Zeitschrift für Gerontologie und Geriatrie, 36(6), 419-420.

Kruse, A., Rentsch, Th., \& Zimmermann, H.-P. (Hrsg.). (2012). Gutes Leben im hohen Alter: Das Altern in seinen Entwicklungsmöglichkeiten und Entwicklungsgrenzen verstehen. Heidelberg: Akademische Verlagsgesellschaft.

Lawton, M. P., Haitsma, K. van, \& Klapper, J. (1996). Observed affect in nursing home residents with Alzheimer's disease. Journal of Gerontology 51(B), 3-14. 
Mantovani, E., \& Turnheim, B. (2016). Navigating the European landscape of ageing and ICT: Policy, governance, and the role of ethics. In E. Domínguez-Rué \& L. Nierling (Hrsg.), Ageing and technology: Perspectives from the social sciences (S. 227-255). transcript.

Murray, E., Burns, J., May, C., Finch, T., O’Donnell, C., Wallace, P., \& Mair, F. (2011). Why is it difficult to implement e-health initiatives? A qualitative study. Implementation Science, 6(6), $1-11$.

Nagel, S. K., \& Remmers, H. (2012). Self-perception and self-determination in surveillance conditions. The American Journal of Bioethics, 12(9), 53-55.

Nussbaum, M. (2011). Creating capabilities. The human development approach. Cambridge: Harvard University Press.

Oleson, F. (2006). Technological mediation and embodied health-care practices. In E. Selinger (Hrsg.), Postphenomenology: A critical companion to ihde (S. 231-247). Albany: State University of New York Press.

Palm, E. (2014). A declaration of healthy dependence: The case of home care. Health Care Analysis, 22(4), 385-404.

Peek, S., Wouters, E., Hoof, J. van, Luikx, K., Boeije, H., \& Vrijhoef, H. (2014). Factors in-fluencing acceptance of technology for aging in place: A systematic review. International Journal of Medical Informatics, 2014(83), 235-248.

Pols, J. (2010). The heart of the matter: About good nursing and telecare. Health Care Analysis, $18(4), 374-388$.

Pols, J. (2012). Care at a distance. On the closeness of technology. Amsterdam: Amsterdam University Press.

Rammert, W. (2003). Technik in Aktion: Verteiltes Handeln in soziotechnischen Konstellationen. In T. Christaller \& J. Wehner (Hrsg.), Autonome Maschinen (S. 289-315). Wiesbaden: Westdeutscher Verlag.

Remmers, H., \& Hülsken-Giesler, M. (2011). e-Health Technologies in home care nursing: Recent survey results and subsequent ethical issues. In M. Ziefle \& C. Röcker (Hrsg.), Human-centered design of e-health technologies. Concepts, methods and applications (S. 154-178). Hershey: Medical Information Science Reference (IGI Global).

Remmers, H., \& Hülsken-Giesler, M. (2012). Kreativität im Alter und die Bedeutung assistiver Technologien - Eine rehabilitationswissenschaftliche Perspektive. In A. Kruse (Hrsg.), Kreativität und Medien im Alter (S. 127-153). Heidelberg: Universitätsverlag Winter.

Remmers, H. (2010). Environments for ageing, assistive technology and self-determination: Ethical perspectives. Informatics for Health and Social Care (IHSC). Special Issue „Ageing and Technology“. December 2010, 35(4), 236-246.

Remmers, H. (2015). Natürlichkeit und Künstlichkeit. Zur Analyse und Bewertung von Technik in der Pflege des Menschen. Technikfolgenabschätzung - Theorie und Praxis. Hrsg. v. Institut für Technikfolgenabschätzung und Systemanalyse, 24(2), 11-20.

Remmers, H. (2016). Ethische Implikationen der Nutzung altersgerechter technischer Assistenzsysteme. Expertise zum Siebten Altenbericht der Bundesregierung. https://www.siebter-altenbericht.de/expertisen-zum-siebten-altenbericht/index.php?eID $=t x \_s e c u r e d o w n l o a d s \& p=36 \& u$ $=0 \& \mathrm{~g}=0 \& \mathrm{t}=1518476604 \&$ hash $=746 \mathrm{ebea} 4 \mathrm{e} 1 \mathrm{e} 023 \mathrm{cdf} 06 \mathrm{ea} 11 \mathrm{~d} 2 \mathrm{e} 729 \mathrm{~cd} 5 \mathrm{ce} 97767 \&$ file $=/$ file admin/altenbericht/pdf/Expertise_Remmers.pdf. Zugegriffen: 11. Febr. 2018.

Robinson, H., MacDonald, B., \& Broadbent, E. (2015). Physiological effects of a companion robot on blood pressure of older people in residential care facility: A pilot study. Australasian Journal on Ageing, 34(1), 27-32.

Saczynski, J. S., Pfeifer, L. A., Masaki, K., Korf, E. S. C., Laurin, D., White, L., \& Launer, L. J. (2006). The effect of social engagement on incident dementia: The Honolulu-Asia aging study. American Journal of Epidemiology, 163(5), 433-440. 
Saito, T., Shibata, T., Wada, K., \& Tanie, K. (2003). Relationship between interaction with the mental commit robot and change of stress reaction of the elderly. Proceedings of the IEEE. International symposium on computational intelligence in robotics and automation. Kobe, S. 119-124.

Sandel, M. J. (1998). Liberalism and the limits of justice (2. Aufl.). Cambridge University Press.

Sävenstedt, S., Sandman, S. O., \& Zingmark, K. (2006). The duality in using information and communication technology in elder care. Journal of Advanced Nursing, 56(1), 17-25.

Sharkey, N., \& Sharkey, A. (2012a). The eldercare factory. Gerontology, 58(3), 282-288.

Sharkey, A., \& Sharkey, N. (2012b). Granny and the robots. Ethical issues in robot care for the elderly. Ethics and Information Technology, 14(1), 27-40.

Siep, L. (2004). Konkrete Ethik. Grundlagen der Natur- und Kulturethik. Frankfurt a. M.: Suhrkamp.

Simon R., Garthaus M., Koppenburger A., \& Remmers H. (2018). Dorfgemeinschaft 2.0 - Altern und Digitalisierung im ländlichen Raum. Zur Entwicklung eines Instruments zur ethischen Fallbesprechung in der ambulanten Gesundheitsversorgung. In M. Pfannstiel, S. Krammer, \& W. Swoboda (Hrsg.) (2018), Digitalisierung von Dienstleistungen im Gesundheitswesen (S. 293-315). Wiesbaden: Springer.

Simon, A., \& Nauck, F. (2013). Patientenautonomie in der klinischen Praxis. In C. Wiesemann \& A. Simon (Hrsg.), Patientenautonomie. Theoretische Grundlagen, praktische Anwendungen (S. 167-179). Münster: Mentis.

Sorell, T., \& Draper, H. (2014). Robot carers, ethics, and older people. Ethics and Information Technology, 16(3), 183-195.

Sorell, T., \& Draper, H. (2012). Telecare, surveillance and the welfare state. The American Journal of Bioethics, 12(9), 36-44.

Sparrow, R., \& Sparrow, L. (2006). In the hands of machines? The future of aged care. Minds and Machines, 16, 141-161.

Sparrow, R. (2002). The march of the robot dogs. Ethics and Information Technology, 4(4), 305318.

Strauss, A., Fagerhaugh, S., Suczek, B., \& Wiener, C. (1980). Gefühlsarbeit. Ein Beitrag zur Arbeits- und Berufssoziologie. In Kölner Zeitschrift für Soziologie und Sozialpsychologie (Bd. 32, S. 629-665).

Sung, H. C., Chang, S. M., Chin, M. Y., \& Lee, W. L. (2015). Robot-assisted therapy for improving social interactions and activity participation among institutionalized older adults: A pilot study. Asia-Pacific Psychiatry: Official Journal of the Pacific Rim College of Psychiatrists, 7(1), 1-6.

Szebehely, M., \& Trydegard, G.-B. (2012). Home care for older people in Sweden: A universal model in transition. Health and Social Care in the Community, 20(3), 300-309.

Valenti Soler, M., Aguera-Ortiz, L., Olazaran Rodriguez, J., Mendoza Rebolledo, C., Perez Munoz, A., Rodriguez Perez, I., Osa Ruiz, E., Barrios Sanchez, A., Herrero Cano, V., Carrasco Chillon, L., Felipe Ruiz, S., Lopez Alvarez, J., Leon Salas, B., Canas Plaza, J. M., Martin Rico, F., Abella Dago, G., \& Martinez Martin, P. (2015). Social robots in advanced dementia. Frontiers in Aging Neuroscience, 7, 133.

Dyk, S. van (2009). 'Junge Alte' im Spannungsfeld von liberaler Aktivierung, Ageism und Anti-Aging-Strategien. In Dyk, S. van \& S. Lessenich (Hrsg.), Die jungen Alten. Analysen einer neuen Sozialfigur (S. 316-339). Frankfurt a. M.: Campus.

Wada, K., Shibata, T., Musha, T., \& Kimura, S. (2008). Robot therapy for elders affected by dementia. IEEE Engineering in Medicine and Biology Magazine, 27(4), 53-60.

Waldenfels, B. (1994). Antwort-Register. Frankfurt a. M.: Suhrkamp.

Weishaupt, S. (2006). Subjektivierendes Arbeitshandeln in der Altenpflege - Die Interaktion mit dem Körper. In F. Böhle \& J. Glaser (Hrsg.), Arbeit in der Interaktion - Interaktion als Arbeit. Arbeitsorganisation und Interaktionsarbeit in der Dienstleistung (S. 85-106). Wiesbaden. 
Whitby, B. (2012). Do you want a robot lover? The ethics of caring technologies. In P. Lin, K. Abney, \& G. A. Bekey (Hrsg.), Robot ethics. The ethical and social implications of robotics (S. 233-248). Cambridge.

Winnicott, D. W. (1953). Transitional objects and transitional phenomena. International Journal of Psycho-Analysis, 34, 88-97.

Zunzunegui, M.-V., Alvarado, B. E., Del Ser, T., \& Otero, A. (2003). Social networks, social integration, and social engagement determine cognitive decline in community-dwelling Spanish older adults. Journals of Gerontology Series B: Psychological Sciences and Social Sciences, 58(2), 93-100.

Hartmut Remmers, Prof. Dr. phil. habil., Universität Osnabrück, Fachbereich Humanwissenschaften, Institut für Gesundheitsforschung und Bildung, Leiter der Abteilung Pflegewissenschaft.

Prof. Remmers war nach einem Studium der Philosophie, Geschichte, Soziologie, Sozialpsychologie und Germanistik (Göttingen und Hannover) sowie Promotion in den Instituten für Soziologie und Politikwissenschaft sowie im Forschungszentrum Zeitgeschichte von Bildung und Wissenschaft der Leibniz-Universität Hannover tätig. An der Universität Bremen hat er als wissenschaftlicher Assistent den ersten berufsbildenden Lehramtsstudiengang Pflegewissenschaft in Deutschland mit aufgebaut. Nach seiner dortigen Habilitation wechselte er an die Universität Osnabrück und leitet dort als Professor die Arbeitsgruppe Pflegewissenschaft. Zu seinen Arbeitsund Forschungsschwerpunkten gehören: Onkologische Pflege, Palliative Care, Alter und Technik, theoretische Grundlagen pflegerischen Handelns, Ethik im Gesundheitswesen.

Das Interesse an Fragen moderner Technologien durchzieht seinen gesamten wissenschaftlichen Werdegang. Bereits in seiner Dissertation über den Soziologen Hans Freyer spielten Fragen der Technik als prägender Faktor moderner Industriegesellschaften (Technokratie-These) eine zentrale Rolle. In den letzten beiden Jahrzehnten führte die Engführung von gerontologischer Pflegeforschung und Entwicklung technischer Assistenzsysteme zu Fragestellungen, die wiederum ins Zentrum philosophischer Grundlagenforschung führen: der theoretischen Explikation und ethisch-normativen Bewertung und Regulation künstlicher Lebensformen.

Open Access Dieses Kapitel wird unter der Creative Commons Namensnennung 4.0 International Lizenz (http://creativecommons.org/licenses/by/4.0/deed.de) veröffentlicht, welche die Nutzung, Vervielfältigung, Bearbeitung, Verbreitung und Wiedergabe in jeglichem Medium und Format erlaubt, sofern Sie den/die ursprünglichen Autor(en) und die Quelle ordnungsgemäß nennen, einen Link zur Creative Commons Lizenz beifügen und angeben, ob Änderungen vorgenommen wurden.

Die in diesem Kapitel enthaltenen Bilder und sonstiges Drittmaterial unterliegen ebenfalls der genannten Creative Commons Lizenz, sofern sich aus der Abbildungslegende nichts anderes ergibt. Sofern das betreffende Material nicht unter der genannten Creative Commons Lizenz steht und die betreffende Handlung nicht nach gesetzlichen Vorschriften erlaubt ist, ist für die oben aufgeführten Weiterverwendungen des Materials die Einwilligung des jeweiligen Rechteinhabers einzuholen.

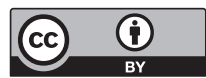

